\title{
Erratum - Classificação pós-tratamento para lesões condrais acetabulares na artroscopia de quadril
}

\section{Post-Treatment Classification for Acetabular Chondral Lesions in Hip Arthroscopy}

Giancarlo Cavalli Polesello ${ }^{1}$ Roxana Viamont-Guerra ${ }^{1}$ Fábio Zego ${ }^{1}$ Nayra Deise dos Anjos Rabelo ${ }^{1,2}$ Walter Ricioli Junior ${ }^{1}$ Marcelo Cavalheiro Queiroz ${ }^{1}$

\footnotetext{
${ }^{1}$ Grupo de Quadril, Departamento de Ortopedia e Traumatologia, Faculdade de Ciências Médicas da Santa Casa de São Paulo (FCMSCSP), Irmandade Santa Casa de Misericórdia, São Paulo, SP, Brasil

2 Laboratório de Análise do Movimento Humano, Departamento de Ciências da Reabilitação, Universidade Nove de Julho - UNINOVE, São Paulo, SP, Brasil
}

Rev Bras Ortop 2019;54:e1.

\section{ERRATUM}

Rio de Janeiro, September 25, 2019

Dear readers,

In the Article Classificação pós-tratamento para lesões condrais acetabulares na artroscopia de quadril* (Rev Bras Ortop DOI: 10.1055/s-0039-1693049.), published online in Revista Brasileira de Ortopedia in August 2019, where it reads:

Roxana Viamont-Guerra ${ }^{1}$

It should read:

Maria-Roxana Viamont-Guerra ${ }^{1}$ de Ortopedia e Traumatologia. Published by Thieme Revinter Publicações Ltda, Rio de Janeiro, Brazil 